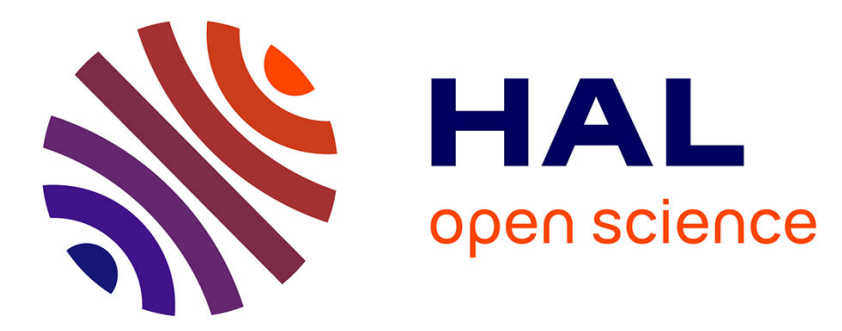

\title{
CDMA-OFDM combination method for enhancing capacity of voice over LTE uplink in PMR context
}

Manh Cuong Nguyen, Hang Nguyen, Eric Georgeaux, Philippe Mège, Laurent Martinod

\section{- To cite this version:}

Manh Cuong Nguyen, Hang Nguyen, Eric Georgeaux, Philippe Mège, Laurent Martinod. CDMAOFDM combination method for enhancing capacity of voice over LTE uplink in PMR context. WCNC 2015: Wireless Communications and Networking Conference, Mar 2015, Nouvelle Orléans, La, United States. pp.658 - 662 10.1109/WCNC.2015.7127548 . hal-01262416

\section{HAL Id: hal-01262416 https://hal.science/hal-01262416}

Submitted on 26 Jan 2016

HAL is a multi-disciplinary open access archive for the deposit and dissemination of scientific research documents, whether they are published or not. The documents may come from teaching and research institutions in France or abroad, or from public or private research centers.
L'archive ouverte pluridisciplinaire HAL, est destinée au dépôt et à la diffusion de documents scientifiques de niveau recherche, publiés ou non, émanant des établissements d'enseignement et de recherche français ou étrangers, des laboratoires publics ou privés. 


\title{
CDMA-OFDM Combination Method for Enhancing Capacity of Voice over LTE Uplink in PMR context
}

\author{
Manh-Cuong Nguyen ${ }^{1}$, Hang Nguyen ${ }^{1}$, Eric Georgeaux ${ }^{2}$, Philippe Mege $^{2}$ and Laurent Martinod ${ }^{2}$
}

\begin{abstract}
In this paper, we introduce a new Code Division Multiple Access (CDMA)-Orthogonal Frequency-Division Multiplexing (OFDM) combination method for Enhancing Capacity of Voice over LTE (VoLTE) Uplink in PMR context. In this method, voice payloads of different User Equipments (UEs) having the same Modulation and Coding Scheme (MCS) can be spread by different orthogonal codes and mapped to the same set of resource elements. The selection of spreading factor allows a maximum reduction of the difference between the LTE Uplink packet size and PMR voice payload. The proposed method allows reducing both data overhead and control overhead issues for VoLTE uplink in PMR context. The CDMA-OFDM combination method gives a significant increasing in capacity of VoLTE uplink over PMR context. In the best case, the proposed method can improve the voice capacity by the factor of 7 in comparison with the standard LTE.
\end{abstract}

Index Terms-CDMA, OFDM, CDMA-OFDM combination, Voice over LTE (VoLTE), Wireless Multicast, Multi-users multiplexing, PMR, user plane

\section{INTRODUCTION}

Professional or Private Mobile Radio (PMR) networks are mainly designed for emergency services like police forces, security services, etc. Current PMR systems are still based on $2 \mathrm{G}$ networks [1]. In order to satisfy the user demands in the future, the PMR network has to evolve to the broadband solutions. In the current broadband technologies, Long Term Evolution (LTE) standard is considered one of the potential candidates for the next generation of PMR. However, the current LTE standards do not have a significant increase of voice capacity in comparison to existing $2 \mathrm{G}$ and $3 \mathrm{G}$ PMR networks. So, the PMR providers are not yet convinced to apply LTE for 4G PMR. The lack of control packets for resource allocation (control overhead) and the difference in packet size between LTE packet size and PMR voice payload are the main obstacles in improving voice capacity of VoLTE in PMR context. Several attempts have been made to enhance voice capacity in LTE (e.g.[2],[3],[4]...). However, previous studies have not dealt with the difference between the LTE packet size and the very small voice payload in case of using LTE for PMR context.

Our research [5] found that the LTE layer one resource allocation is not efficient and not suitable for small voice

\footnotetext{
${ }^{1}$ Institut Mines-Telecom, Telecom SudParis Samovar Laboratory, UMR 5157, CNRS, Evry, France $\{$ manh_cuong.nguyen, hang.nguyen $\} @$ it-sudparis.eu

${ }^{2}$ Department of Security and Communication Solutions CASSIDIAN (an EADS Company), Elancourt, France \{eric.georgeaux, philippe.mege, laurent.martinod\}@ cassidian.com This work was accomplished in the framework of the collaborative project SOAPS.2, labeled by the Systematic PARIS-REGION Cluster
}

packets. Regarding the resource allocation strategy, one pair of Physical Resource Blocks (PRBs) is the smallest User Assignment Unit in LTE. The smallest LTE packet size is still too large in case that low bit rate voice communication is transmitted in high quality channel. The gap between LTE packet size and the small voice payload reduces significantly the voice capacity of the system. To improve the voice capacity for VoLTE in PMR context, in [6], we have introduced a Multiusers Multiplexing Radio Voice Transmission method for the downlink transmission. To provide a complete solution, in this paper, we propose a new CDMA-OFDM combination method for enhancing voice capacity over LTE uplink in PMR context. In our method, voice packets from different users having the same MCS values can be spread by different orthogonal codes before being mapped to the same set of resource elements. The selection of spreading factor allows a maximum reduction of the difference between the LTE Uplink packet size and PMR voice payload. In addition, we also apply a new RNTI aggregation method to reduce the control overhead for VoLTE in PMR context. Therefore, the proposed method allows reducing both data overhead and control overhead issues for VoLTE uplink in PMR context. The paper is organized as follows. Subsection II-A describes the architecture for CDMA-OFDM combination method. Subsection II-B describes in details the proposed algorithm for determining the spreading factor, code assignment and resource allocation. Section II-D presents the proposed scheduling for CDMA-OFDM combination method. The performance of our method is evaluated in section III. Section IV gives the conclusion and some perspectives.

\section{PROPOSED CDMA-OFDM COMBINATION METHOD}

\section{A. Proposed CDMA-OFDM Combination Architecture}

1) Sender side: The proposed architecture for spreading VoLTE uplink is presented in Fig. 1. In this architecture, data bits from MAC layer (MAC PDUs) of Voice UE after adding the Cyclic redundancy check (CRC), channel coding, will be modulated and spread by an orthogonal sequence. Then, the OFDM symbols undergo layer mapping. In our method, packets of UEs in a spreading group will be code-multiplexed and mapped into the same set of resource elements (REs).

The spreading code, which is used for the voice packets, is the Orthogonal Variable Spreading Factor (OVSF) codes. The using of OVSF allows from 4 to 512 different terminals in the same cell can transmit simultaneously (see Fig. 2). The spreading factor will be chosen based on the bandwidth of LTE uplink, Modulation and Coding Scheme of UE, and the 


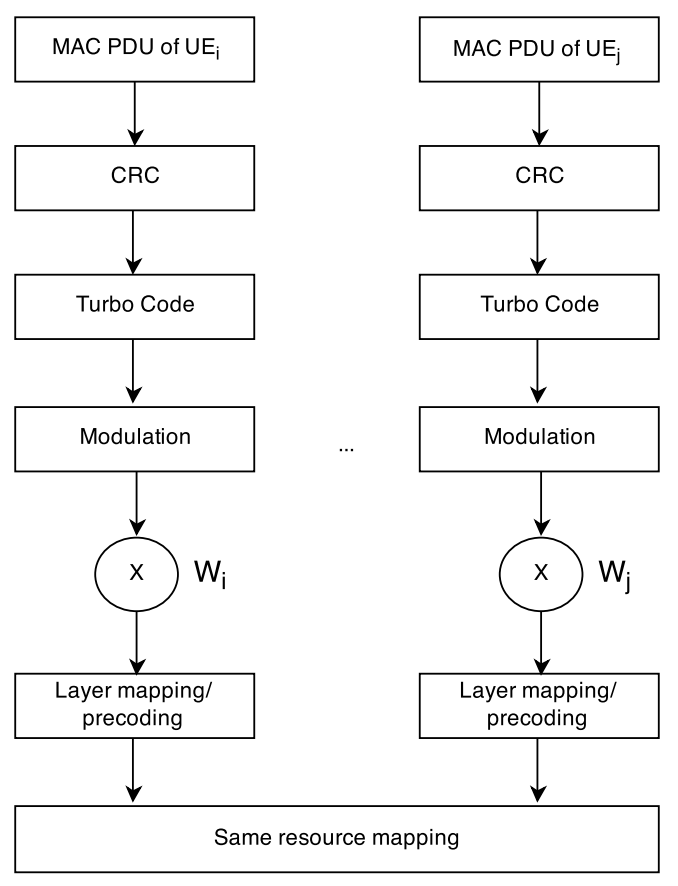

Fig. 1. Architecture of using CDMA-OFDM combination in LTE uplink

voice payload of UE. Algorithm for scheduling and assign the Spreading Code for each UE will be discussed in II-B.

The idea of combining OFDM and CDMA, which is called multi-carrier code division multiple access (MC-CDMA) has been studied in [7], [8]. The researches showed that this combination has number of advantages. In MC-CDMA, signal can be easily transmitted and received using FFT device without increasing the transmitter and receiver complexities. MC-CDMA retains the frequency-diversity of CDMA but robust to channel frequency selectivity. MC-CDMA allows using the available spectrum efficiently. We apply the MCCDMA in case of VoLTE Uplink to increase the voice capacity in the PMR context.

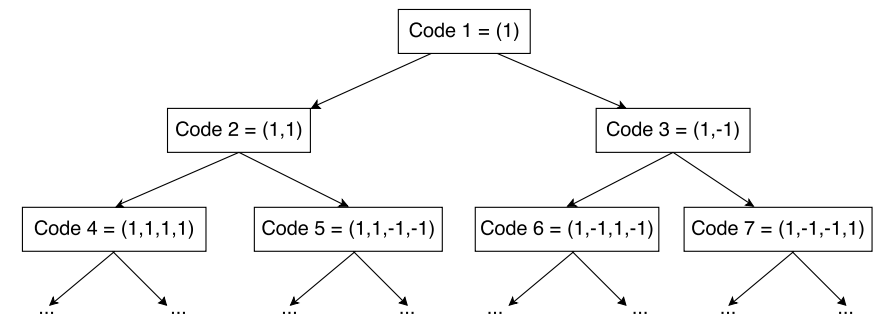

Fig. 2. OVSF code tree for CDMA-OFDM method

2) Receiver side: The eNodeB processes the received signal as follows. Firtly, it removes the cyclic prefix, performs the DFT, equalization and despreading. Next, eNodeB uses the Demodulation reference signals (DMRS) to demodulate the Physical Uplink Shared Channel (PUSCH). In our case, we have to modify the DMRS design to adapt the CMDA-OFDM

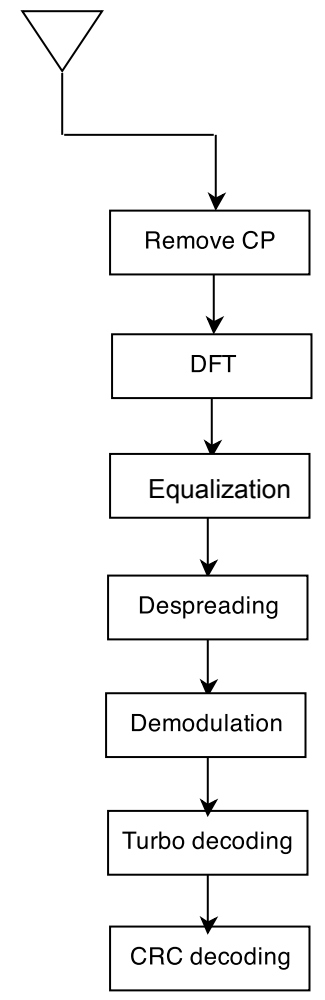

Fig. 3. CDMA-OFDM architecture at eNodeB

method. The details of adaptation for DMRS design will be presented in II-C. Then, eNodeB process the channel decoding, $\mathrm{CRC}$ decoding to reconstruct the MAC PDUs.

$B$. Proposed spreading factor determination, resource allocation and code assignment algorithm in the CDMA-OFDM combination method

For the calculation of the spreading factor and the voice capacity, we made some adaptations of the algorithm presented in articles [5] and [6].

Voice packets is emitted by the Application Layer with size of $T_{\text {voice }}$. These packets are added the header each time passing the real-time transport protocol (RTP), user datagram protocol (UDP) and IP for transmission. Next the voice packets are compressed at the Packet Data Convergence Protocol (DPCP) layer by the Robust Header Compression (ROHC). Then the PDCP header, RLC header and Mac header are added to create their payload, $T B S_{\text {temp }}=P_{\text {voice }}+H_{\text {overhead }}$ The output packets of MAC layer will undergo the Cyclic Redundancy Check, channel coding and be modulated. The output symbols of modulation component will be spread by an orthogonal code. In our method, eNodeB determines the spreading factor $S F$ based on the TBStemp, the number of allowed resource blocks of the defined bandwidth $\left(N_{B W}\right)$ and the MCS. Then, eNodeB will perform the scheduling and send resource allocation information and the code assignment information for UE Uplink in the Physical Downlink Control Channel (PDCCH) channel. 


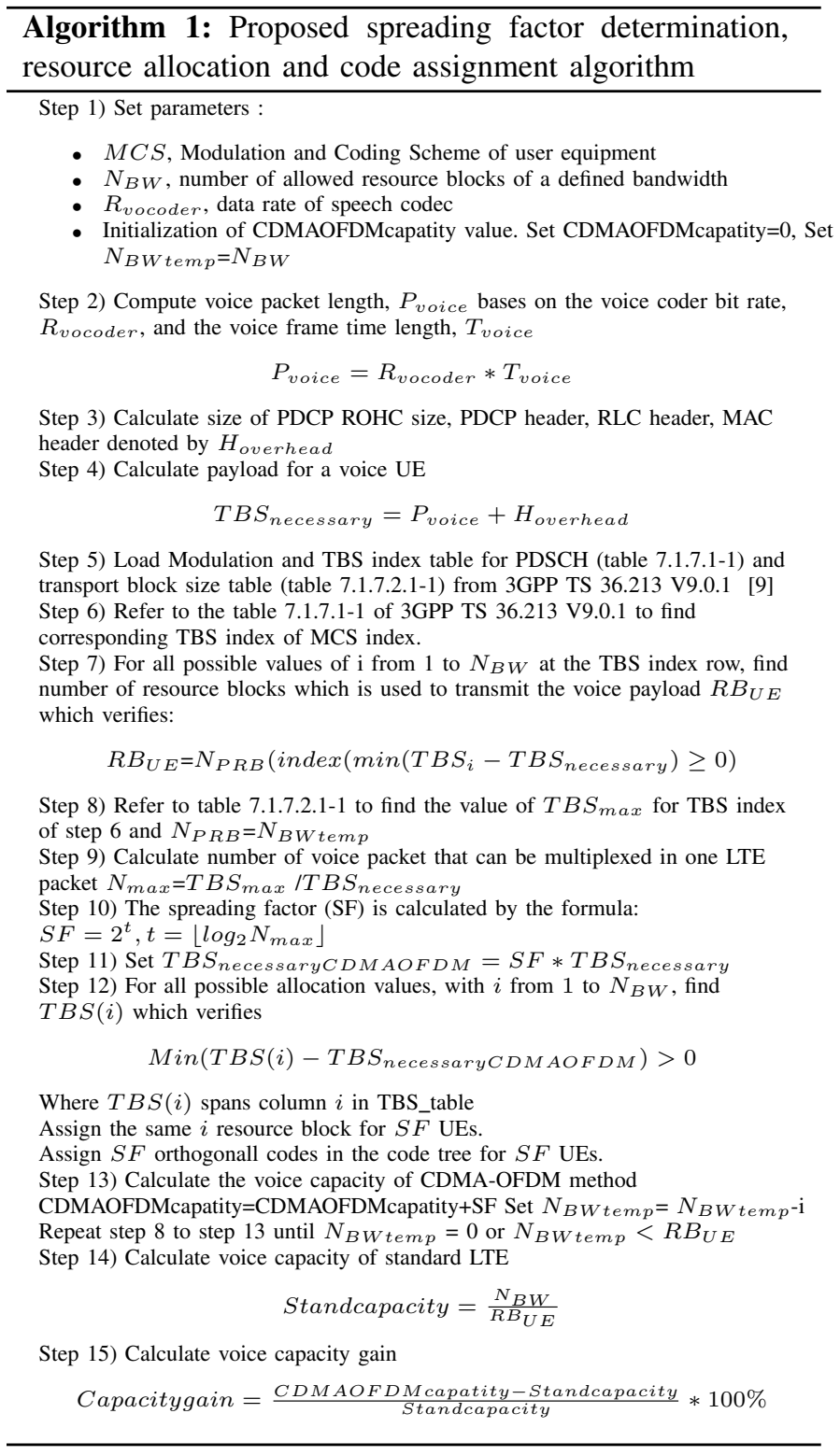

\section{Proposed DMRS Design for CDMA-OFDM Combination}

Demodulation reference signals (DMRS) is used by the base station for channel estimation for coherent demodulation of the uplink channels. DMRS uses the $4^{\text {th }}$ SC-FDMA symbols in each slot (LTE frame type 1). Each UE uses different DMRS sequences. DMRS sequence $r_{\alpha}^{u, v}(n)$ is given by:

$$
r_{\alpha}^{u, v}(n)=e^{j \alpha n} \bar{r}_{u, v}(n), 0 \leq n<M_{R S}^{s c}
$$

where:

- $\alpha$ : cyclic shift (CS) value

- $M_{R S}^{s c}$ : length of DMRS sequence

- $\bar{r}_{u, v}(n)$ : base sequence

In order to support the demodulation of packets from several UEs that are spread in one LTE packet, the DMRS design must be adapted to support the CDMA-OFDM combination method. We propose to apply and adapt method of [10].
Assume that at the $i^{t h}$ TTI, the CDMA-OFDM Combination method clusters $n_{S F}$ UEs for spreading. In our method, UEs in a same spreading group will use the same base sequence $\bar{r}_{u, v}(n)$. The CS value of DMRS for $i^{t h}$ UE in the multiplexing group will be calculated by formula 2 :

$$
\begin{gathered}
\alpha_{i}=2 \pi n_{c s, i} / 12 \\
n_{c s, i}=\left(n_{c s, 0}+\frac{C}{n_{S F}} . i\right) \bmod (C), i=0,1, . ., n_{S F}-1
\end{gathered}
$$

Where:

- $n_{c s, i}$ : CS value of DMRS for $i^{t h} \mathrm{UE}$

- $n_{c s, 0}$ : CS value for the first UE in the group.

- $C$ : constant value 12

In our method, the DMRS sequences of the UEs in the same spreading group are orthogonal. This allows the eNodeB to separate from each other the received demodulation signals of the different UEs in the same spreading group.

\section{Proposed CDMA-OFDM Combination Scheduling}

In LTE, for the transmission of Downlink (DL) and Uplink (UL) transport channels, associated control signaling have to be used. LTE uses Physical Downlink Control Channel (PDCCH) to carry all allocation information for both downlink and uplink shared channels. PDCCH can use first one to three OFDM symbols in each subframe to carry Downlink Control Information (DCI). DCI carries the information about Resource block assignment, Modulation and coding scheme (MCS) some other additional information such as Transmit Power Control (TPC) command for PUCCH, HARQ process number... In standard LTE, each PDCCH contains different resource allocation information for each UE. In our case, the group classifier and group selector will classify and select voice UEs to send to MAC scheduler. The criteria for classifying the UEs is MCS values of UEs. UEs having the same MCS values will be put in a same group. In our scheduling, PDCCHs of a spreading group contains the same information about Resource Block Assignment (RBA) and MCS for the group. UEs in a same group are distinguished by the code position field in the DCI (see Table I).

TABLE I

DCI FORMAT FOR THE CDMA-OFDM COMBINATION SYSTEM

\begin{tabular}{|l|l|l|}
\hline Field Name & Length (bits) & Comment \\
\hline \multirow{2}{*}{ RBA } & $3(1.4 \mathrm{Mhz})$ & \\
& $5(3 \mathrm{Mhz})$ & Same for all UEs in the group \\
& $7(5 \mathrm{Mhz})$ & \\
\hline MCS & 5 & Same for all UEs in the group \\
\hline \multirow{3}{*}{ Code position } & $4(1.4 \mathrm{Mhz})$ & \\
& $6(3 \mathrm{Mhz})$ & Different for each UE \\
& $8(5 \mathrm{Mhz})$ & \\
\hline TPC for PUSCH & 2 & Power control \\
\hline CS for DMRS & 3 & See Table 5.5.2.1.1-1 in 36.211 \\
\hline
\end{tabular}

To reduce the control overhead issue that occurs when there is not enough PDCCH channels for the resource allocation for the CDMA-OFDM combination method. We proposed a new RNTI aggregation method. This method uses PDCCH channel 
with high format, created by the aggregation-of PDCCH with low format, to transmit several RNTIs of different UEs in a same group (see Fig.5). The number of RNTI in one PDCCH is calculated to ensure that there is no increase of Bit Error Rate (BER) for receiving PDCCH channel. The value of code position in the DCI is the base value for determining the code position of each UE in the PDCCH. Assume that there are $k$ RNTIs aggregated in one PDCCH channel and the value of code position in the DCI is $p$. So the code position of UE having the $i^{t h} R N T I$ in the PDCCH is $p+k-i$ (Figure 4). In the limited scope of this article, we do not go into the details of our RNTI aggregation method.

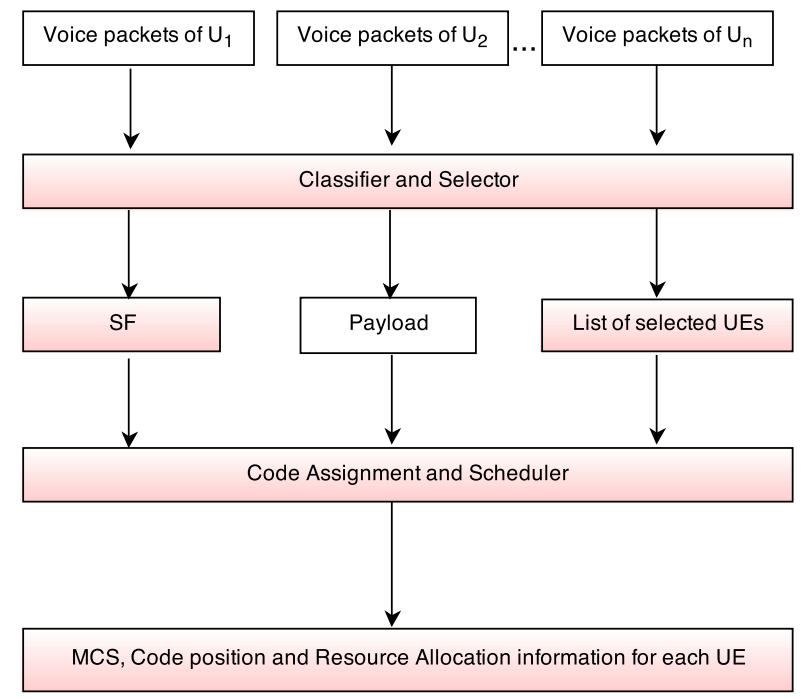

Fig. 5. CDMA-OFDM scheduling

\section{Performance Evaluation}

In our context, the voice capacity of LTE is evaluated with PMR constraints so that we use a very low bit rate voice coder (Advanced Multiband Excitation 2450 bps codec). The evaluation is computed for three deployment bandwidths (1.4 $\mathrm{MHz}, 3 \mathrm{MHz}, 5 \mathrm{MHz}$ ) for Uplink Transmission. Table II provides the main simulation parameters.

TABLE II

SIMULATION PARAMETERS

\begin{tabular}{|l|l|}
\hline Parameter & Value \\
\hline Bandwidth & $1.4 \mathrm{MHz}, 3 \mathrm{MHz}, 5 \mathrm{MHz}$ \\
\hline LTE & Type 1 FDD \\
\hline Codec & AMBE 2450 bps \\
\hline Symbols for PDCCH & 3 symbols \\
\hline Ng & 1 \\
\hline CRC & 24 bits \\
\hline Cyclic Prefix & Normal \\
\hline CCEs for Uplink & $50 \%$ CCEs \\
\hline Number of antenna & 2 \\
\hline
\end{tabular}

A comparison of the voice capacity between the proposed method and the standard LTE is presented in Figure 6. As shown in Figure 6, the proposed method reported significantly more voice capacity than the standard LTE for $20 \mathrm{~ms}$ evaluation of the $1.4 \mathrm{MHz}$ bandwidths. We can find that the efficiency of the algorithm increases with MCS. This is because the scheduling mechanism in LTE is not optimized for small voice payload and the standard does not offer a reasonable choice for a very small voice payload. When the channel quality is high (higher MCS), the number of bits transmitted in a PRB can increase rapidly due to the selection of higher-order modulation and high turbo code rate. However, one pair of PRBs can transmit maximum one voice payload from one UE. In our case, when the LTE packet size is expanded, we can transmit voice payloads from several UEs by a flexible spreading size therefore we can get higher voice capacity gain. In the best case (MCS value equal to 28 and LTE bandwidth equal $5 \mathrm{MHz}$ ), the proposed method can multiply the voice capacity by the factor of 7 . Figure 7 and 8 present the voice capacity gain of our method in terms of number of users for $20 \mathrm{~ms}$ evaluation for $3 \mathrm{MHz}$ and $5 \mathrm{MHz}$ bandwidths. With the same value of MCS, the larger the bandwidth, the higher the voice capacity gain our method can get. Table III illustrates the average control capacity gain of the proposed method. From the data in Table III, it is apparent that the proposed method also allows a significant reduction of the control overhead. The proposed method allows reducing both data overhead and control overhead issues for VoLTE uplink in PMR context.

TABLE III

CONTROL CAPACITY GAIN OF THE PROPOSED METHOD FOR $20 \mathrm{~ms}$

\begin{tabular}{|l|l|l|l|}
\hline Bandwidth & $1.4 \mathrm{MHz}$ & $3 \mathrm{MHz}$ & $5 \mathrm{MHz}$ \\
\hline PDCCH capacity of FD & 40 & 120 & 200 \\
\hline PDCCH capacity of new method & 60 & 240 & 460 \\
\hline Average control capacity gain & $50 \%$ & $100 \%$ & $130 \%$ \\
\hline
\end{tabular}

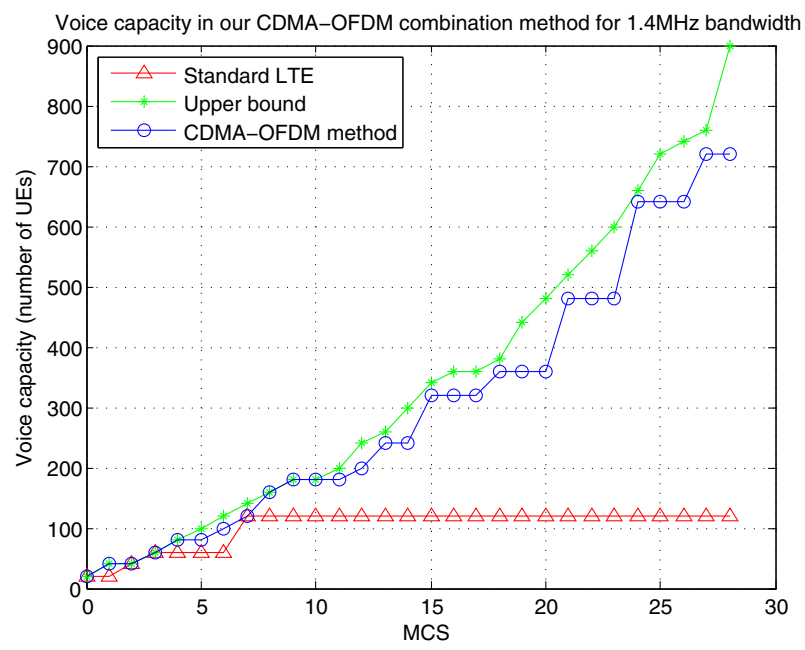

Fig. 6. Voice capacity gain of the proposed method for the $1.4 \mathrm{MHz}$ bandwidth for $20 \mathrm{~ms}$

\section{CONCLUSION}

In this paper, we discussed and evaluated a new CDMAOFDM combination method that aims to improve the uplink 

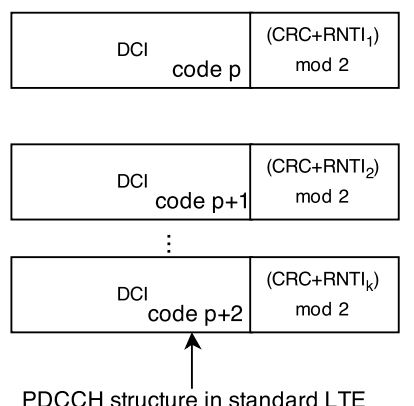

code $p+k$
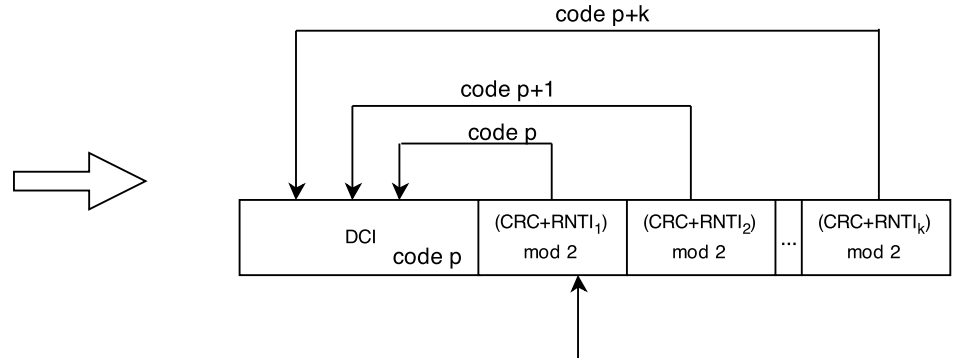

PDCCH structure in the proposed method

Fig. 4. PDCCH structure in the proposed method

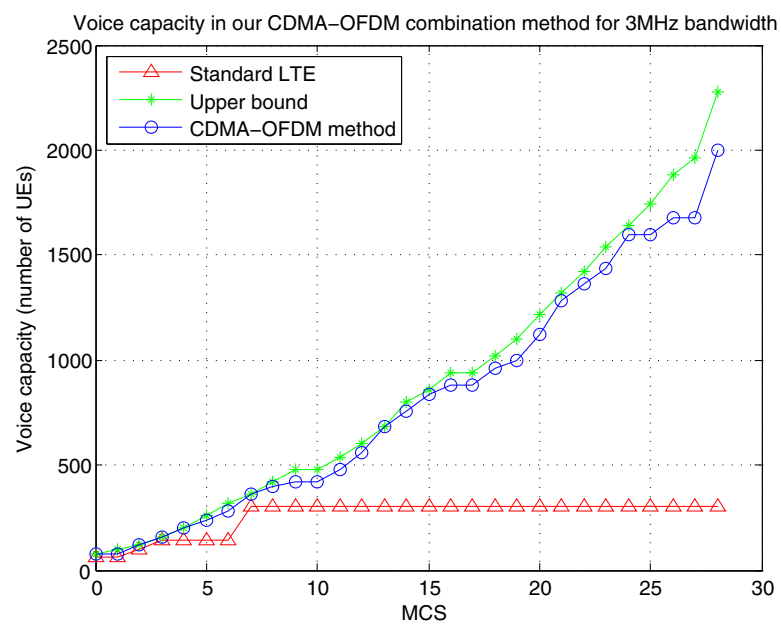

Fig. 7. Voice capacity gain of the proposed method for the $3 \mathrm{MHz}$ bandwidth for $20 \mathrm{~ms}$

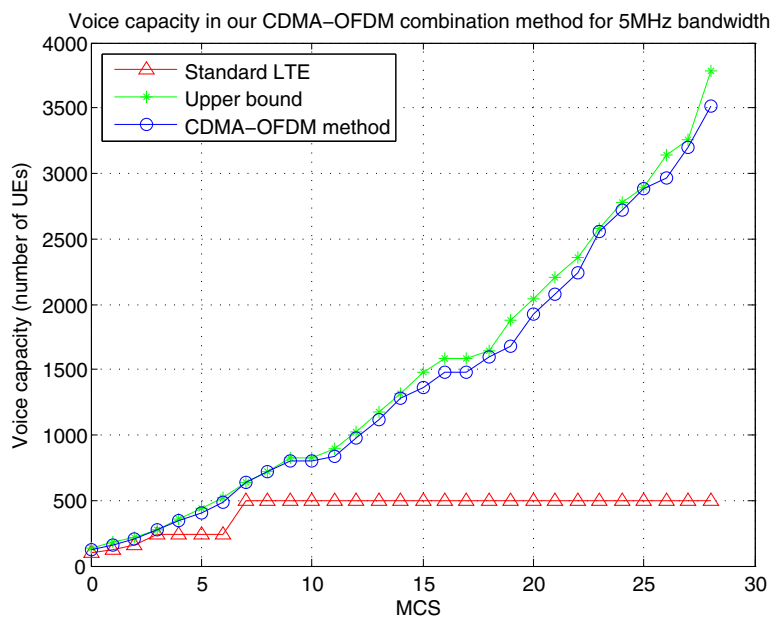

Fig. 8. Voice capacity gain of the proposed method for the $5 \mathrm{MHz}$ bandwidth for $20 \mathrm{~ms}$

voice capacity of VoLTE in PMR context. In our method, the voice payload of different UEs having the same MCS can be spread by different orthogonal codes and mapped to the same set of resource elements. The spreading factor is chosen for maximum reduction of the difference between the LTE Uplink packet size and PMR voice payload. The lack of control signaling is solved by a RNTI aggregation mechanism. In addition, we also proposed a new DMRS design and a new scheduling scheme for the CDMA-OFDM combination method. The results indicate that our proposition gives a significant increasing in capacity of VoLTE uplink over PMR context. In the best case, the proposed method can multiply the voice capacity by the factor of 7 in comparison with the standard LTE. However, this research has raised up many questions in need of further investigation. First, power control issue needs to be considered. Second, phase and frequency synchronization issue has to be addressed.

\section{REFERENCES}

[1] XP Masbernat, C Gruet, F Fraysse, S Contal, and L Naviner. Green solutions for future lte pmr networks. In Computer Aided Modeling and Design of Communication Links and Networks (CAMAD), 2012 IEEE 17th International Workshop.

[2] J Han and H Wang. Principle and performance of tti bundling for voip in lte fdd mode. In Wireless Communications and Networking Conference, 2009. WCNC 2009. IEEE.

[3] H Wang and D Jiang. Performance comparison of control-less scheduling policies for voip in te ul. In Wireless Communications and Networking Conference, 2008. WCNC 2008. IEEE.

[4] D Jiang, Haiming Wang, Esa Malkamaki, and Esa Tuomaala. Principle and performance of semi-persistent scheduling for voip in lte system. In Wireless Communications, Networking and Mobile Computing, 2007. WiCom 2007. International Conference.

[5] AA Florea, L Martinod, P Mege, and H Nguyen. Multi-layer realistic voice capacity evaluation in lte rel. 9 and performance comparison with pmr and gsm. In World Telecommunications Congress (WTC), 2012.

[6] MC Nguyen, H Nguyen, E Georgeaux, P Mege, L Martinot, and A Florea. Architecture for Multi-users Multiplexing Radio Voice Transmission for Enhancing Voice Capacity over LTE in PMR Context. Personal, Indoor and Mobile Radio Communications (PIMRC), 2014.

[7] Shinsuke Hara and Ramjee Prasad. Design and performance of multicarrier cdma system in frequency-selective rayleigh fading channels. Vehicular Technology, IEEE Transactions, 1999.

[8] S Tomoki and F Adachi. Comparative study of various frequency equalization techniques for downlink of a wireless ofdm-cdma system. IEICE transactions on communications, 2003.

[9] Evolved Universal Terrestrial Radio Access (E-UTRA); Physical layer procedures, 3GPP TS. 36.213 V9.0.1, Dec. 2009.

[10] Xiaolin Hou, Zhan Zhang, and Hidetoshi Kayama. Dmrs design and channel estimation for lte-advanced mimo uplink. In Vehicular Technology Conference Fall (VTC 2009-Fall), 2009 IEEE 70th. 